\title{
Estrategia artística para mejorar la expresión oral en estudiantes mexicanos: un estudio piloto
}

\author{
Artistic strategies to improve oral production in Mexican \\ students: a pilot study
}

DOI: https://doi.org/10.32870/dse.v0i23.779

Tomás Jesús Gómez Calles*

Higinio Fernández-Sánchez ${ }^{* *}$

Hercy Baez Cruz***

Mariana Pérez-Pérez ${ }^{* * * *}$

\section{Resumen}

La presente investigación tiene como objetivo realizar un estudio piloto para, posteriormente, llevar a cabo una intervención didáctica mediante actividades de escultura y modelado de plastilina para favorecer la expresión oral en alumnos de educación primaria. Para ello se realizó la selección de un grupo muestra conformado por 28 niños en edades de 6 a 8 años, con quienes se trabajó una técnica artística denominada "Escultura Familiar", para que a través ella comuniquen la percepción y los sentimientos que experimentan con respecto a su familia. La metodología utilizada para este estudio piloto corresponde a un paradigma cuantitativo con un diseño pre-experimental y la aplicación de un pretest-postest que permitió, a partir del uso de la estrategia señalada, que los alumnos desarrollaran sus habilidades manuales y aumentaran el nivel de expresión oral al exponer y argumentar sus comentarios apoyados en su escultura. Los resultados de este estudio muestran que la intervención es prometedora, y que podría potenciar y estimular el nivel de expresión oral en los alumnos, corroborando que esta resultó ser una gran herramienta educativa. Asimismo, da pauta para implementar la intervención a mayor escala, donde se pueda evaluar la eficacia y la efectividad.

Palabras clave: artes - escultura familiar - estudio piloto - expresión oral - modelado.

\section{Abstract}

The aim of this research was to implement a didactic intervention through clay modeling and sculpture activities to favor oral expression in elementary school students. A sample group of 28 children aged 6 to 8 years employed an artistic technique known as "family sculpture" to communicate their perceptions

\footnotetext{
* Licenciado en Educación Primaria. Director y docente del nivel. Licenciado en Gastronomía y licencia técnica en servicios turísticos. gomezcalles@hotmail.com

** Maestro en Enfermería y candidato provisional al Doctorado en Enfermería, Universidad de Alberta. higinio@ualberta.ca

*** Doctor en Educación. Ingeniero en Electrónica y maestrías en Educación y Administración. hercyb@hotmail.com

**** Estudiante de la Licenciatura en Enfermería. Universidad Veracruzana, Campus Veracruz. México. mperezperez299@gmail.com
} 
and feelings regarding their family. The methodology used for this pilot study, following a quantitative paradigm with a pre-experimental design and the application of a pretest-posttest, allowed us to deploy the aforementioned strategy to help the students develop their manual skills and oral expression by supporting their arguments about their sculptures. Our preliminary findings revealed that this intervention is promising and that it might enhance and stimulate the level of oral expression in elementary school students, as well as inform future intervention work at a larger scale to assess its efficacy and effectivity.

Keywords: arts - family sculpture - pilot study - oral expression - modeling.

\section{Introducción}

La Escultura Familiar (EF) como medio artístico puede ayudar a aumentar la expresión oral en estudiantes de primaria. La EF forma parte de la Terapia en Artes Expresivas (TAE) y busca que las personas exterioricen la personalidad de cada miembro de su familia a través de figuras realistas elaboradas con plastilina (Malchiodi, 2012). La expresión oral se entiende como la capacidad de una persona para hablar y expresarse, teniendo como meta ser entendido (Castro, 2013); no obstante, la expresión oral requiere de herramientas de apoyo como la EF para ser más eficiente.

Los estudiantes de primaria, en especial los de primer grado, se encuentran en una etapa de desarrollo lingüístico que incluye la expresión oral. De acuerdo con los estadios propuestos por la Teoría del Desarrollo Cognitivo de Jean Piaget, los educandos en el primer año de educación primaria se ubican dentro de la fase preoperacional (de 2 a 7 años) (Miller, 2011). Dicha etapa es aquella donde los escolares construyen experiencias sobre el mundo, mediante la adaptación, hasta lograr la consolidación de un pensamiento lógico. Esquinas y Sánchez (2011) mencionan que se debe tener una formación integral en la educación primaria, basándose en la transversalidad de aspectos formativos; la plástica ofrece un medio ideal para la transmisión de mensajes formativos transversales.

En México, se ha visto un déficit en la expresión oral en niños menores de 10 años; según datos estadísticos del Instituto Nacional para la Evaluación de la Educación (INEE, 2014), los estudiantes de tercer grado de preescolar únicamente alcanzan el nivel básico de expresión oral. Por ejemplo, $87 \%$ de los niños que egresan de la educación preescolar y escuchan una narración, pueden exponer lo que escucharon y lo que provoca en ellos mismos; sin embargo, solo 50\% logra dar una explicación coherente en su narración. En 2018, la Secretaría de Educación Pública (SEP) reportó más de 2'000,000 de alumnos inscritos en el sexto grado de primaria, de los cuales al menos $50 \%$ presentaron un déficit en el lenguaje y la comunicación (Ramos, 2018).

Por lo antes expuesto, surge la necesidad de enriquecer la expresión oral con actividades e intervenciones que propicien un aumento en su nivel de desarrollo. De acuerdo con Cardona y Celis (2011), es necesario complementar las clases con intervenciones diversificadas en otras asignaturas, como el caso del uso de estrategias artísticas, a fin de contribuir al desarrollo de la comunicación y expresión verbal de los estudiantes de educación primaria. De tal modo que 
los docentes requieren considerar importante el hecho de enriquecer la expresión oral en los niños, con la intención de que logren el desempeño de sus competencias comunicativas acorde a su desarrollo cognitivo y a los aprendizajes esperados que se encuentran establecidos en los programas educativos vigentes.

En Perú, Rozas (2018) realizó una intervención artística al utilizar el karaoke como estrategia para mejorar la expresión oral, presentando un avance significativo en los estudiantes; En tanto, Alfaro (2009) encontró que la dramatización, como medio artístico, logró mejorar la fluidez y coherencia, así como aumentar el nivel de expresión oral en estudiantes que participaron en esta intervención. Es así como las estrategias artísticas para mejorar la expresión oral han demostrado ser efectivas. Monsalva y Rodríguez (2016) exponen que existe una dificultad para expresarse en situaciones de comunicación real de forma espontánea, de ahí la necesidad de construir actividades lúdicas que permitan al alumno mejorar su confianza y seguridad, y a su vez les permita crear situaciones comunicativas que fortalezcan sus habilidades orales. Sánchez (2016) señala que las artes plásticas aplicadas con niños de nivel primaria ayudan a desarrollar la competencia comunicativa, ya que son actividades agradables para los alumnos además de que fomentan su interés.

Green (2013) menciona que las expresiones artísticas deben usarse como estrategias para favorecer el lenguaje y la comunicación de los estudiantes ya que permiten expresar sentimientos, estados anímicos e ideas con libertad e imaginación, reacciones con respecto al mundo que los rodea, creencias filosóficas y religiosas, conceptos sobre el orden y los conflictos universales. Las estrategias de apoyo consisten en hacer una revisión sobre actividades artísticas que generen soluciones para favorecer y aumentar el nivel de expresión oral en los alumnos de educación primaria.

Basados en la información presentada, se puede observar que el déficit de expresión oral en estudiantes de primer grado de primaria representa una barrera para la correcta comunicación con los demás. Derivado de esta área de oportunidad, surge la necesidad de realizar intervenciones con actividades artísticas ya que, en la edad en que se encuentran los estudiantes del primer año (7 años) su imaginación está en niveles altos, mismos que deben ser aprovechados para desarrollar y elevar el nivel de este tipo de expresión.

\section{Metodología}

\section{Diseño}

El presente estudio piloto tiene un enfoque cuantitativo. Se utilizó un diseño pre-experimental con un grupo de intervención y mediciones pretest y postest; este diseño permitió disponer de un punto de referencia inicial, a fin de analizar el nivel que tiene una muestra de estudio respecto a las variables dependientes antes de aplicar el estímulo, lo cual permite llevar un seguimiento del progreso de dicho grupo de estudio (Cruz, Olivares, González, 2014; León, Davis, 
Kraemer, 2011; Shadish, Cook, Campbell, 2002). En este sentido, la capacidad de expresión oral como variable del estudio se midió antes y después de la intervención.

Dada la naturaleza del estudio piloto, no se requiere un cálculo de tamaño para la muestra, aunque varios autores recomiendan no sea menor a 10 o 12 participantes, puesto que este tipo de estudio es uno de los primeros pasos para lograr desarrollar una intervención más ambiciosa (Fernández, Guzmán, Herrera et al. 2020). Cabe mencionar que la efectividad y eficacia no son el enfoque de los estudios piloto. Por lo contrario, se recomienda que el análisis sea de tipo descriptivo.

Otro punto para considerar es la experiencia del interventor en temas similares a los que comprende la intervención, ya que esto permite una mayor fluidez y dominio de las actividades planeadas. En este sentido, el interventor o persona que realizó la estrategia cuenta con una licenciatura en educación primaria.

\section{Participantes y contexto}

Para el desarrollo de la investigación piloto se identificó y reclutó un grupo de alumnos de primer grado de una escuela primaria rural federalizada, en el municipio de Tuxpan, Veracruz. La muestra total fue de 28 participantes, con un rango de edad entre 6 y 8 años, en su mayoría, pertenecen a una comunidad rural clasificada como una localidad de clase social baja, y la mayor parte de los padres de familia se dedican a actividades agrícolas y ganaderas.

Para la realización del estudio, en un primer acercamiento se tuvo contacto con el director de la escuela; en esta reunión se le expusieron: 1) los objetivos de la investigación; 2) el planteamiento de la participación de los estudiantes; 3 ) los beneficios para los educandos y la investigación; y finalmente, 4) se solicitó una segunda reunión, ahora con los padres de familia y los estudiantes, donde se les dio a conocer la información antes mencionada. Asimismo, en el caso de que aceptaran participar, se les requeriría firmar una carta de consentimiento informado, tanto a ellos como a los estudiantes.

\section{Componente}

De acuerdo con Olivares, Macià, Olivares y Roza (2012), la investigación tuvo una intervención unicomponente, ya que los sujetos de estudio presentaban en común la dificultad para expresarse oralmente y para atender el problema, se hizo uso de la escultura familiar como estrategia de intervención a partir de actividades de escultura y modelado con plastilina, para aumentar la capacidad de expresión oral en los estudiantes considerados como muestra, utilizando a la familia como eje central.

\section{Dosis}

La intervención se llevó a cabo en tres sesiones de dos horas, los días 23, 24 y 25 de junio de 2019, en horario de las 10 a 12 horas.

Diálopos sobre Educación 


\section{Intervención}

\section{Sesión 1}

La primera actividad se llevó a cabo en cuatro momentos: en los primeros 15 minutos el facilitador se presentó y realizó una dinámica de relajación, a fin de generar un ambiente de confianza. La actividad consistió en que los participantes movieran el cuerpo al compás de música de jazz. Después, se sentaron en el piso formando un semicírculo, y se les pidió que cerraran los ojos y pensaran en los integrantes de su familia por 15 minutos. Enseguida, cada uno de los participantes pasaron al frente del aula a compartir las características de cada una de las personas que conforman su familia, así como el tiempo de convivencia con cada uno de ellos. Cada estudiante tuvo tres minutos para compartir la información con el resto de los participantes, dando un total de 84 minutos. En esta sesión, se observó y registró el vocabulario y desenvolvimiento oral de los participantes. Durante los últimos 11 minutos de la sesión, el facilitador proporcionó un aperitivo que consistió en fruta picada y un vaso de agua fresca de fruta, mientras se hicieron comentarios sobre los puntos de vista de la actividad realizada en esta sesión.

\section{Sesión 2}

La segunda sesión se organizó en tres momentos. El primero, y con la finalidad de generar un ambiente armonioso, los participantes se sentaron en el suelo tomando una postura cómoda, cerraron los ojos y se concentraron en el ritmo de su respiración (15 min.) mientras escuchaban música relajante. A continuación, el facilitador entregó a cada uno de los participantes una caja con barras de plastilina de diversos colores y papel cascarón. En los 90 minutos siguientes los estudiantes elaboraron una escultura que representaba a cada integrante de su familia. Mediante la escultura, cada uno de ellos debía demostrar las características individuales de todos sus familiares. El participante inició con su propia representación, jerarquizando de mayor a menor y de izquierda a derecha las esculturas que elaboró, según la trascendencia en su vida. Finalmente, en los 15 minutos restantes de la sesión, el facilitador proporcionó un pequeño snack que consistió en barritas de granola y agua natural.

\section{Sesión 3}

La tercera sesión también se desarrolló en tres momentos. En los primeros 15 minutos, se llevó a cabo la misma actividad de la segunda sesión, para integrar a los participantes. En la segunda parte, cada integrante del grupo de estudio expuso de manera oral, por tres minutos, su escultura frente al grupo. Utilizando su escultura familiar, los participantes describieron las características de cada uno de los integrantes de su familia (ej. nombre, aspecto físico y personalidad). Además, el participante explicó el motivo del orden en el que fueron colocadas las esculturas, haciendo énfasis en la persona más cercana y la más distante a él. Finalmente, en los 11 minutos restantes de la sesión, el facilitador agradeció el tiempo y disposición de los participantes para realizar esta intervención y entregó a cada uno un kit escolar. 


\section{Efectos preliminares}

Las mediciones se realizaron antes y después de la intervención. Los efectos preliminares se midieron mediante una rúbrica analítica que determinó el desempeño de la expresión oral. La rúbrica fue elaborada por el primer autor del presente estudio, específicamente para esta intervención. Consta de cuatro indicadores en los cuales el participante: 1) reconoce a los integrantes de su familia; 2 ) identifica las características de sus familiares (i.e. nombre, aspecto físico y personalidad); 3) utiliza el tiempo establecido para hacer su presentación; por último, 4) expresa de manera clara y precisa las características de los integrantes de su familia. La rúbrica fue evaluada considerando los siguientes niveles de desempeño: 1) no lo hace; 2) lo hace con dificultad; 3) lo hace bien; y finalmente, 4) lo hace excelente. Las sumas de los valores obtenidos determinaron el nivel de expresión oral; nivel bajo (4-8), nivel medio (9-12) y nivel alto (13 o más).

Para complementar los efectos preliminares, se presentan algunas narrativas de los participantes en el pre y post test. Es importante mencionar que estas narrativas se derivan de componentes propios de la intervención, donde los participantes tuvieron la oportunidad de describir a los miembros de su familia. Es aquí donde se rescataron comparaciones entre las respuestas que dieron a las preguntas: “Cómo es tu familia? / ¿Podrías describir a tu familia?". Para estos registros, las preguntas se hicieron en la medición pretest y postest. Las narrativas se registraron mediante notas en una libreta de seguimiento.

\section{Análisis}

Los datos se analizaron utilizando la estadística descriptiva e inferencial. Las variables categóricas (i.e. género) se representaron en frecuencias y porcentajes, y las variables continuas (i.e. edad) se expresaron mediante medidas de tendencia central. Asimismo, se realizó la prueba Shapiro Wilk para verificar normalidad en la distribución de los datos. La prueba arrojó normalidad ( $p>.000)$, por lo que se procedió a realizar la prueba T-student, utilizada en datos paramétricos. Para el análisis de datos se utilizó el programa SPSS para Windows 10.

\section{Consideraciones éticas}

El trabajo fue revisado y aprobado por el comité de ética e investigación del Centro Regional de Educación Normal (CREN) Dr. Gonzalo Aguirre Beltrán. Asimismo, acatando las normas internacionales y nacionales en términos de investigación con seres humanos, se utilizó el consentimiento informado por escrito, tanto de los padres como de los niños. En él se establecía que el participante aceptaba ser parte de la investigación; sin embargo, si aceptaba en un inicio y decidía abandonar la intervención, era libre de hacerlo.

\section{Resultados}

\section{Características de los participantes}

La tabla 1 muestra el total de participantes $(n=28), 67.8 \%$ son del sexo masculino, con una edad promedio de 7 años y un rango entre 6 y 8 años. $39.2 \%$ de los participantes provienen de famil- 
ias nucleares, mientras que $32.1 \%$ se ubica en familias monoparentales y $28.5 \%$ corresponde a familias de padres separados. Se identificó que el nivel socioeconómico de los participantes corresponde a los niveles: medio (39.2\%) y bajo (60.8\%).

Tabla 1. Datos sociodemográficos

\begin{tabular}{lcc}
\hline \multicolumn{1}{c}{ Variable } & $f$ & $\%$ \\
\hline \multicolumn{1}{c}{ Género } & & \\
Masculino & 9 & 32 \\
Femenino & 19 & 67.2 \\
Composición Familiar & & \\
Nuclear & 11 & 39.2 \\
Monoparental & 9 & 32.1 \\
Padres separados & 8 & 28.5 \\
Nivel socioeconómico & & \\
Alto & 0 & 0 \\
Medio & 11 & 39.2 \\
Bajo & 17 & 60.8 \\
\hline Fuente: Cuestionario de Datos Sociodemográficos $\mathrm{n}=28$.
\end{tabular}

\section{Tasa de participación y deserción}

De los 34 participantes iniciales que cumplían con los criterios de inclusión, 32 (padres e hijos) dieron el consentimiento informado por escrito para participar (94.1\% tasa de participación), y 28 (hijos) terminaron el estudio (87.5\% tasa de participación). Un participante abandonó la intervención debido a un cambio de institución educativa (3.1\% tasa de deserción); cuatro participantes no obtuvieron el consentimiento de los padres (12.5\%) y un participante no mostró agrado por las actividades manuales (3.1\%).

\section{Asistencia y participación}

Los participantes debían asistir a tres sesiones, de las cuales se obtuvo un rango de asistencia que oscila entre $92 \%$ y $100 \%$ (tabla 2 ).

Tabla 2. Asistencia a las sesiones

\begin{tabular}{ccc}
\hline Sesión & $f$ & $\%$ \\
\hline 1 & 29 & 100 \\
2 & 28 & 92.8 \\
3 & 28 & 92.8 \\
\hline
\end{tabular}

Fuente: Lista de Asistencia $\mathrm{n}=28$. 


\section{Resultados preliminares}

En la tabla 3 se exponen los resultados de la evaluación pretest y postest, en la primera se señala que $92.9 \%$ de los participantes presentaban un nivel bajo de expresión oral, y después de la intervención se observó que $57.1 \%$ de los participantes alcanzó el nivel medio. Estos datos demostraron cambios significativos en la expresión oral, antes $(M=5.42 ; D S=1.67)$ y después $(M=$ 9.07; $\mathrm{DS}=1.84$ ) de la implementación de la intervención $t(27), \mathrm{T}=-12.479, p=.000$.

Tabla 3. Resultados de evaluaciones

\begin{tabular}{|c|c|c|c|c|}
\hline \multirow[b]{2}{*}{ Nivel de Expresión Oral } & \multicolumn{2}{|c|}{$\begin{array}{c}\text { Resultados } \\
\text { Pretest }\end{array}$} & \multicolumn{2}{|c|}{$\begin{array}{c}\text { Resultados } \\
\text { Postest }\end{array}$} \\
\hline & $f$ & $\%$ & $f$ & $\%$ \\
\hline Nivel Bajo de Expresión Oral & 26 & 92.9 & 11 & $39 \cdot 3$ \\
\hline Nivel Medio de Expresión Oral & 2 & 7.1 & 16 & 57.1 \\
\hline Nivel Alto de Expresión Oral & 0 & o & 1 & 3.6 \\
\hline Total & 28 & 100.0 & 28 & 100.0 \\
\hline
\end{tabular}

Fuente: Escala de Medición de la Expresión Oral n = 28 .

\section{Resultados complementarios}

A continuación, presentamos algunas descripciones que ofrecieron los participantes antes de la intervención:

P1: "mi familia es bonita"; "mi mamá es chiquita y mi papá grandote"; "yo soy feliz".

P11:"en mi familia somos felices, mi mamá mi abuelita y yo".

P21: "pues en mi casa solo vivimos mi mamá yo".

P28: "vivimos mi tía, mi tío, mi abuelita, mi abuelito, mis tres hermanos, mis papás y yo".

Tras la intervención, estas son algunas descripciones que hicieron los mismos participantes:

P1: Mi familia es grande, somos 5; mi mamá que es pequeña, delgada, su pelo chino y es café igual que sus ojos, ella es muy alegre; mi papá es alto, gordito, tiene poco cabello negro y es muy gruñón, le gusta usar camisas de cuadros; mi hermana " $x$ " es más grande que yo, jugamos siempre juntas, ella usa dos trenzas que le hace mi mamá, es flaquita y muy alegre; mi hermanito " $x$ " es más pequeño que yo, el aún usa pañal y mamila, le gusta dormir mucho y aquí estoy yo, soy pequeña pero no tanto, ya voy a la escuela, me gusta jugar mucho con mis amigos, me gusta usar mi fleco y también tenis para correr rápido".

P11: Esta es mi familia, mi papá nos abandonó cuando era bebé, entonces se vino a la casa mi abuelita, ella tiene el pelo blanco, usa lentes y cocina bien rico, siempre me hace el lonche para la escuela, juega 
conmigo y me lleva y me trae a la escuela porque mi mamá trabaja para que podamos tener cosas en la casa. Mi mamá es bonita, es muy trabajadora, le gusta usar pantalón de mezclilla y tenis, siempre se peina para atrás con una liga y cuando llega tarde del trabajo me abraza, me pregunta que cómo me fue en la escuela y revisa mi tarea. Nomás somos tres, pero somos bien felices en la casa.

P21: Uy maestro, pues mi familia somos mi mamá yo. Ella tempranito me levanta en la mañana y me hace de desayunar, me trae a la escuela y se va a trabajar. Trabaja en una oficina de secretaria, siempre va muy arreglada, ella es muy linda, tiene los ojos cafés, el cabello lacio y siempre usa sus aretes de perlita. Yo soy este, aunque soy chaparrito y llenito, soy muy alegre, me gusta contar chistes y hacer reír a mis amigos.

P28: Mi familia si es muy grande, vivimos muchos en mi casa; viven mi tío X y mi tía X, mi tío es panzón pero muy alegre, siempre arregla cosas en la casa y me hace juguetes de madera; mi tía es gruñona, le molesta que mi tío tome, ella siempre me ayuda con la tarea y va por mí a veces a la escuela; mis abuelitos son chistosos, ya tienen el pelo blanco y la cara como pasitas, ya son viejitos, siempre duermen y les gusta estar viendo la tele. Mi mamá y mi papá se van a trabajar a la tienda que tienen, mi mamá siempre se levanta temprano y hace el desayuno para todos, mi papá a veces le ayuda, pero él siempre abre la tienda. Son muy cariñosos conmigo y mis tres hermanos, son dos hermanas y un hermano. Nosotros siempre jugamos en el patio de atrás, nos gusta andar descalzos porque hace siempre mucho calor.

\section{Discusión}

Los resultados de esta investigación sugieren que favorecer la expresión oral en niños utilizando la técnica de la escultura familiar a partir de actividades artísticas, permite abrir nuevas posibilidades de comunicación en torno a situaciones que viven los infantes en distintos contextos en los que se desenvuelven. De modo que a partir de los hallazgos antes expuestos, se detectaron tres áreas de oportunidad: la práctica docente, las políticas de educación y futuros proyectos de investigación.

Con los resultados obtenidos se demuestra que como estrategia artística, la escultura familiar logró aumentar el nivel de expresión oral en los niños considerados en la muestra, lo cual coincide con otras investigaciones en las que se hizo uso de diversas estrategias artísticas para elevar la expresión oral en estudiantes, tales como el uso del karaoke (Rozas, 2018), la dramatización (Alfaro, 2009), el trabajo con las artes plásticas (Sánchez, 2016), así como el uso de la expresión plástica (Dionisio, 2017). Por lo anterior, se sugiere que la intervención pueda realizarse a mayor escala en futuros proyectos de investigación para que permitan medir su efectividad y eficacia; adicionalmente, se recomienda capacitar a los docentes en el uso e implementación de diversas estrategias artísticas, a fin de que sean capaces de diversificar y trabajar varias herramientas con fines educativos en el aula. 
Cabe señalar que los participantes en el presente estudio mostraron interés en las actividades de intervención, lo cual se vio reflejado en el índice de aceptación y, con ello, en la disponibilidad para concluir las sesiones; en este caso podría señalarse que fueron varios los factores que influyeron en la respuesta de los niños, y uno de ellos pudo ser el uso de estrategias iniciales de relajación a partir de la música pues, como señala Gómez, Viger y Cantero (2013), el uso de esta estrategia permite descargar tensiones y generar una atmósfera de bienestar; así también, los estimulantes al cierre de las clases, la relación que se establece entre las actividades y el entorno familiar de los infantes. Como señalan Quintana y Aparicio (2017), los estudiantes aprenden y recuerdan mejor la información cuando esta se contextualiza con su entorno familiar, y ello les permite disponer de la confianza para hablar de un tema que es de su total dominio, como lo es la relación con su familia. Por lo tanto, se recomienda que toda intervención didáctica busque vincularse con los entornos y contextos en donde se desenvuelven los estudiantes, además de que el docente en todo momento genere un ambiente de aprendizaje armónico y de confianza para favorecer en los alumnos su motivación intrínseca para el trabajo a realizar.

El hecho de que no todos los padres de los estudiantes que cumplían con los criterios de inclusión hayan firmado el consentimiento informado fue una limitante durante el proceso de selección de la muestra, dado que no es éticamente posible hacer un estudio con la participación de individuos menores de edad que no están autorizados por sus tutores o que ellos mismos no desean participar en un proceso de investigación que implica hablar de su entorno familiar; tema que pudiera derivar en afectaciones emocionales debido a la situación en la que viven o bien, una invasión a su intimidad familiar. Por tal motivo, se hace necesaria la creación de estrategias de reclutamiento innovadoras que no pierdan de vista el sentido ético al momento de realizar investigaciones con seres humanos, que cuenten con un diseño claro para el destinatario y que disponga de apertura para dejar bien establecidos los propósitos, alcances y limitaciones de la investigación que ha de desarrollarse.

El estudio permitió conocer los efectos preliminares de una intervención artística para aumentar el nivel de expresión oral en un grupo de estudiantes de educación primaria; no obstante, es posible señalar que también se logró identificar algunas limitantes, como el hecho de que al no haber existido un grupo de comparación, no fue posible hacer una evaluación más completa de la estrategia aplicada; adicionalmente, por el hecho de que la muestra fue pequeña en comparación con la población disponible en la escuela. Por tanto, para futuras intervenciones valdría la pena considerar dos grupos distintos a los que se aplique el pretest y postest a fin de valorar los mismos indicadores, pero solo en uno de ellos se aplique la estrategia didáctica de estudio. También se podría sugerir hacer uso de este tipo de estrategias, pero utilizando una metodología cualitativa, a fin de realizar una evaluación que permita complementar los resultados numéricos obtenidos en esta experiencia de investigación. 


\section{Conclusión}

Trabajar una intervención holística a partir de la técnica de escultura familiar permitió favorecer la expresión oral de los niños considerados en la muestra, dado que presentaron un cambio favorable en el nivel de su expresión al término de la intervención; datos que concuerdan con estudios previos. Pese a que los resultados fueron satisfactorios y estadísticamente significativos, es necesario afinar y detallar la intervención para incrementar y mejorar sus alcances. La afinación de esta intervención incluye el aumento de sesiones y la duración de estas, haciendo énfasis en la importancia de aplicar actividades artísticas como estrategia para aumentar el nivel de expresión oral.

La intervención con la técnica de la escultura familiar, desde el momento que involucra el desarrollo de una actividad manual e imaginativa, es prometedora para ampliar el nivel de expresión oral en los participantes, de manera que la diferencia de dichos niveles entre el pretest y postest revelaron ser estadísticamente significativos, pues aproximadamente la mitad de los estudiantes presentaron un aumento en el nivel de oralidad después de la intervención. Esta conclusión es coherente con estudios anteriores que respaldan un resultado positivo en el aumento de la expresión oral como efecto de intervenciones que involucren actividades artísticas. Sin embargo, además deben tomarse en consideración factores externos como el ambiente de aprendizaje establecido por el docente.

Los docentes de la institución se encontraron satisfechos con la intervención, misma que fue adaptada al grado y contexto en el que se aplicó gracias a sus aportaciones; los alumnos mostraron también sorpresa al poder expresar con mayor facilidad sus ideas después de la intervención; asimismo, se mostraron cómodos con el facilitador. Este último es un docente de educación primaria que trabaja con alumnos de este nivel educativo, de allí la facilidad para adaptar la intervención; al estar familiarizado el profesor con las características de los participantes se vio entusiasmado, al igual que los alumnos; esto contribuyó a la satisfacción por la intervención y su óptimo resultado, mismo que debe ser explorado en investigaciones futuras con un mayor número de estudiantes u otros grupos de alumnos.

\section{Referencias}

Alfaro, L. (2009). Técnica de dramatización y su influencia en la expresión oral de los alumnos de segundo grado de la I.E. "Don José de San Martín" de Tacna, 2008. [Tesis]. Perú: Universidad Privada de Tacna.

Cardona, J.; M. Celis (2011). Estrategias para mejorar la expresión oral en el grado cuarto de educación de básica primaria. [Tesis].

https://edudistancia2001.wikispaces.com/file/view/ESTRATEGIAS+PARA+MEJORAR+LA+EXP RESION+ORAL+EN+EL+GRADO+4\%C2\%BO+DE+BASICA+PRIMARIA.pdf 
Cruz del Castillo, C.; S. Olivares; M. González (2014). Metodología de la investigación. México: Patria.

Dionisio Contreras, Y. K. (2017). El fantástico mundo del relato y la expresión plástica: su contribución al desarrollo de los procesos de apropiación de la lectura y la escritura, en niños y niñas de 6 a 12 años de edad en un contexto de diversidad. [Tesis].

http://repository.udistrital.edu.co/bitstream/11349/6747/1/DionisioContrerasYulianaKatherin2017.pdf

Esquinas, F.; M. Sánchez (2011). Didáctica del dibujo: artes plásticas y visuales (3a ed. Vol. II). España: Graó.

Fernández, H., Guzmán, F., Herrera, D., et al. (2020). Importancia del Estudio Piloto en un Proyecto de Intervención. Index de Enfermería.

https://ciberindex.com/index.php/ie/article/view/e12860

Gómez Artiga, A.; P. Viguer Segui; M. J. Cantero López (2013). Intervención temprana. Desarrollo

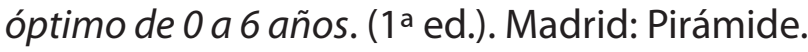

Green Rojas, E. L. J. (2014). Expresiones artísticas como estrategia para el enriquecimiento del lenguaje y la comunicación. [Tesis].

http://biblio3.url.edu.gt/Tesario/2013/05/09/Green-Estela.pdf

Instituto Nacional para la Evaluación de la Educación (2014). Panorama Educativo de México: Indicadores del Sistema Educativo Nacional. México: INEE.

https://www.inee.edu.mx/wp-content/uploads/2019/01/P1B112.pdf

León, A. C.; L. L. Davis; H. C. Kraemer (2011). The Role and Interpretation of Pilot Studies in Clinical Research. Journal of Psychiatric Research, 45(5), 626-629.

https://doi.org/10.1016/j.jpsychires.2010.10.008

Malchiodi, C. A. (2012). The Handbook of Art Therapy. Guilford. https://doi.org/10.1002/9781118306543

Olivares Rodríguez, J.; D. Macià Antón; P. J. Olivares Olivares; A. I. Rosa Alcázar (2012). El ejercicio de la psicología aplicada. La profesión del psicólogo. Madrid: Pirámide.

https://books.google.com.mx/books?id=RLuUBQAAQBAJ\&pg=PA140\&dq=T\%C3\%A9cnicas+de+Intervenci\%C3\%B3n+y+Tratamiento+Psicol\%C3\%B3gico+unicomponente\&hl=es\&sa=X\&ved=0ahUKEwjAhr2qhabpAhVrhq0KHYiiDBsQ6AEIMTAB\#v=onepage \&q=T\%C3\%A9cnicas\%20de\%20Intervenci\%C3\%B3n\%20y\%20Tratamiento\%20Psicol\%C3\%B3gico\%20unicomponente\&f=false

Quintana Albalat J.; O. Y. Aparicio Gómez (2017). Temas emergentes en educación. Bogotá: Universidad Central.

Ramos, M. (2018). Educación y aprendizaje. México: UNICEF. https://www.unicef.org/mexico/educaci\%C3\%B3n-y-aprendizaje

Rozas Huacho, G. (2018). El karaoke como medio para mejorar la comunicación oral de los niños y niñas del nivel primario, de la institución educativa $n^{\circ} 50011$ de Cachona. [Tesis].

http://repositorio.uarm.edu.pe/handle/UNIARM/1573 
Sánchez Vera, E. (2016). Fortalecimiento de habilidades comunicativas a través de las artes plásticas, con niños y niñas de cuarto de primaria de la institución educativa distrital Saludcoop Sur. J. T. Universidad Libre. [Tesis]. https://repository.unilibre.edu.co/handle/10901/9600

Shadish, W.; T. Cook; D. Campbell (2002). Experimental and Quasi-Experimental Designs for Generalized inference. Boston/New York: Houghton Mifflin Company. 